\title{
THE INFLUENCE OF VELOCITY OF LENGTH CHANGE ON TENSION DEVELOPMENT IN SKELETAL MUSCLE: MODEL CALCULATIONS AND EXPERIMENTAL RESULTS
}

\author{
F. A. M. VAN KAAM and E. L. DE BEER \\ Department of Medical Physiology. Slate University of Utreclat \\ and \\ G. J. M. StIEnEN and T. BLANGÉ \\ Department of Physiology, University of Amsterdam
}

\begin{abstract}
Force responses obtained during constant velocity length changes on skeletal muscle tissue are simulated by means of two cross-bridge models proposed by Huxley and Simmons (1971. Nalure 233, 533-538) and by Julian et al. (1974. Biophys. J. 14, 546-562). An implicit method was used for the numerical approximation in the simulations. The simulated force transients due to constant velocity length changes are found to be in qualitative agreement with re-investigated experimental results obtained from the whole sartorius muscle of the frog. A non-linear tension transient is observed, dependent both on amplitude and on velocity of release revealing an inflexion which gives the transient a shoulder shape. When velocity is increased the intlexion occurs carlier and at a lower tension value.

A non-linear transient is observed during stretches performed at moderate velocities. Force responses are found to deviate concavely downwards from a linear time course. Simulations, however, predict a rather linear tension transient for comparable velocities. Implications of the experimental findings are discussed for both models.
\end{abstract}

\section{INTRODUCTION}

Tension transients recorded after quick length changes (Gordon et al., 1966a; Huxley and Simmons, 1971a, b, 1973; Ford et al., 1977, 1981; Julian et al., 1973) or length transients recorded after quick load changes (Podolsky, 1960; Podolsky and Nolan, 1973) are frequently studied to obtain information about the dynamic properties of the contraction mechanism of stimulated skeletal muscle fibres. Huxley and Simmons (1971b) introduced a cross-bridge model (the HSmodel) based on sliding filaments as the element of the contraction mechanism (Huxley, 1953, 1971; Hanson and Huxley, 1953; Huxley and Niedergerke, 1954; Huxley, 1957). This model describes mathematically the fast tension recovery, as measured on single fibres after a quick length change (Huxiey and Simmons, 1971a, b, 1973). The HS-model explains the fast recovery by transition of attached cross-bridges between two (or more) distinct states. However, in this model the final recovery has not been incorporated.

A modification of Huxley's 1957-model (Huxley, 1957) and the HS-model has been proposed by Julian, Sollins and Sollins (further referred to as the JSS model; Julian et al. 1973, 1974). Transitions between two distinct states of attachment as well as between attached and detached states govern the redevelopment. This model describes redevelopment of tension to its isometric value after a quick change in length, including also the slow recovery phase.

Recrived August 1983; in revised form March 1984.
One of the key assumptions in both the HS- and the JSS-model is the linear relationship between tension and extension in the $S_{2}$ link of the cross-bridge, according to Hooke's law. Transition rates between these states were introduced based both on this assumption and on the existence of different potential energy levels in different attached states.

A non-linear relation was found between the extreme tension value $T_{1}$ at the end of the release and the amplitude of the length change (Huxley and Simmons, 1971b. 1973). This non-linearity was attributed to recovery already taking place during the length change (truncation) and was found to increase with length change. The tension recovery during the length change, which in turn is dependent on the velocity of length change, thus affects the tension development after the length change. Even in experiments with an improved time resolution (Ford et al, 1977) considerable truncation occurred within $0.2 \mathrm{~ms}$. Since tension recovery plays an important role during the length change we carried out a simulation of the HS-model as well as the JSS-model in which the duration of the length change was taken into account. The simulations have been calculated numerically for several (constant) velocities of length change.

The calculations at different velocities of length change were compared with re-investigated experimental results obtained from the whole sartorius muscle of the frog. It has already been shown that experiments performed on whole skeletal muscle of the frog agree rather well qualitatively with the results of single fibre experiments (Stienen et al., 1978; Blangé and Stienen, 1979; Stienen and Blangé, 1980, 1981). 
The results of both model simulations and of the whole muscle experiments will be discussed together with the results of similar single fibre experiments obtained from the literature. Some of the results have already been published in abstract form (De Beer et al., 1982).

\section{METHODS}

Experiments were performed on the isometrically contracting sartorius muscle of the frog Rana esculenta, dissected free together with a part of the pelvic bone and a small part of the tibia. At slack length (about $30 \mathrm{~mm}$ ) the muscle was mounted with its pelvic end on the displacement system and its tibial end on the force transducer. Length changes were executed at rest length, i.e. at a sarcomere length of about $2.2 \mu \mathrm{m}$ at which force generation is optimal (Ramsey and Street, 1940; Gordon et al., 1966a).

The bathing fluid, composed of $115 \mathrm{mM} \mathrm{NaCl}$, $2.5 \mathrm{mM} \mathrm{KCl}, 1.8 \mathrm{mM} \mathrm{CaCl}_{2}$ and a sodium phosphate buffer ( $\mathrm{pH}=7.2$ ), was kept at about $2 \mathrm{C}$.

A strain gauge force transducer as described by Blangé et al. (1972) with a resonance frequency of $7 \mathrm{kHz}$ was used to measure tension responses during the quick length changes. The displacement system with an interferometer device described by Stienen et al. (1978) controlled the position and movement of the pelvic end. A supramaximal stimulation (tetanus) was applied by two silver wires running alongside the whole muscle.

Recordings of the output of the displacement system and the force transducer were photographed from a storage oscilloscope (HP 1201 B) and were recorded on tape (Ampex FR 1300).

Further detailed information of the experimental set-up is given by Stienen (1981).

\section{MOdEL CaLCLLATIONS}

For the derivation of the numerical equations in which the velocity of length change is incorporated, the basic equations as described by Huxley and Simmons (1971b, 1973) and by Julian et al. (1974) can be used. The basic principle of both models is given schematically in Fig. 1 together with the rate constants used. $D$ denotes the detached state. $n_{1}$ and $n_{2}$ denote the configurations of the attached states. Rate constants of transitions between attached states $\left(k_{1}, k_{2}\right)$ or attached and detached state $\left(k_{3}, k_{4}\right)$ are given as a function of the total extension $y$ in the $S_{2}$ link of the cross-bridge. The characteristics of both models can be summarized as follows:

Both assume a linear relationship between tension and an instantaneous extension or compression of the $\mathrm{S}_{2}$ link according to Hooke's law.

No attachment or detachment takes place in the HSmodel; only transitions between attached states.

In the JSS-model, attachment only takes place to configuration state $n_{1}$ of cross-bridges with zero extension $\left(k_{4}\right.$ is zero for all $y$ values except $y=0$ ).

In the JSS-model, there is detachment of crossbridges exist in configuration state $n_{2}$.

The difference in extension in the $S_{2}$ link between both configurations of the attached states, $h$, amounts to $8 \mathrm{~nm}$ in the HS-model and to $10 \mathrm{~nm}$ in the JSSmodel.

In the JSS-model, attached cross-bridges exist in the equilibrium isometric situation with extension zero in configuration state $n_{1}$ or with extension $10 \mathrm{~nm}$ in configuration state $n_{2}$. In the HS-model, the extension of the $S_{2}$ link in the same situation amounts to 4 and 12 $(4+h) \mathrm{nm}$ respectively.

In an isometrically contracting muscle, $18 \%$ of the cross-bridges are situated in state $n_{1}$, and $23 \%$ in state $n_{2}$ in the JSS-model and the remaining $59^{\circ}$ is
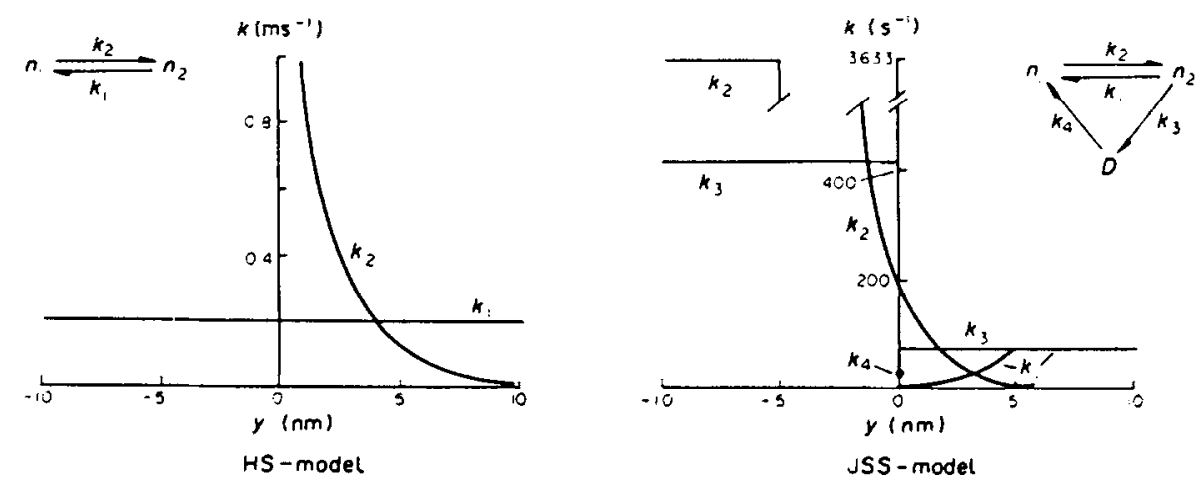

Fig. 1. Simplified schemes of the contraction models and rate functions as described for the HS-model and the JSS-model (Huxley and Simmons, $1971 \mathrm{~b}$; Julian et al., 1974). D, $n_{1}$ and $n_{2}$ denote the relative numbers of cross-bridges in detached state and two configurations of attached states respectively. Rate constants are given as function of the total extension $y(\mathrm{~nm})$ in the $S_{2}$ link of a cross-bridge. In the HS-model $k_{1}$ and $k_{2}$ are expressed as the number of attached cross-bridges relative to the total number of attached cross-bridges that turn over per $\mathrm{ms}$. In the JSS-model transition rates are given as the absolute number of cross-bridges that turn over per second per square centimetre per half sarcomere 
detached. In the HS-model, both configuration states have an equal amount of attached cross-bridges.

In both models full activation (tetanus) and a maximum overlap of the filaments is assumed. This implies that the experiments are limited to the plateau region of the length-tension curve (Gordon et al., 1966b).

Simulations are calculated for a temperature of $4 \mathrm{C}$ for the HS-model and of $0 \mathrm{C}$ for the JSS-model corresponding to the temperatures at which the parameter values and rate constants were available (Huxley and Simmons, 1971b, 1973; Julian et al., 1973. 1974).

After an instantaneous shift of the initial crossbridge distribution the basic equations as derived in the HS- and JSS-models describe the cross-bridge redistribution after the length change. The general cross-bridge distribution for both configuration states at time $t$ is $n_{1}(u, t)$ and $n_{2}(u+h, t)$, where $u$ is the extension of the $S_{2}$ link in the cross-bridge in the undisturbed isometric situation (tetanus) for the attached configuration state $n_{1}$. The extension in state $n_{2}$ thus amounts to $u+h$. A relative filament displacement $x(t)$ is defined as the displacement of the thick filament relative to the adjacent thin filaments. Similarly $x^{\prime}(t)$ is defined as the velocity of the thick filament relative to the adjacent thin filament which is chosen constant in the simulations. The basic equations derived from the schemes as depicted in Fig. 1 as given by the two models for a general cross-bridge distribution $n_{1}(u, l)$ and $n_{2}(u+h, t)$ in an adjusted notation are

for the HS-model (Huxley and Simmons, 1971b; equation 9)

$$
\begin{aligned}
\mathrm{d} n_{2}(u+h+x, t) / \mathrm{d} t & =k_{2}(u+x)-\left[k_{2}(u+x)\right. \\
+ & \left.k_{1}(u+h+x)\right] n_{2}(u+h+x, l) \\
n_{1}(u+x, t) & =1.0-n_{2}(u+h+x, t)
\end{aligned}
$$

for the JSS-model (Julian et al., 1974; equations 1 and 2)

$$
\begin{gathered}
\hat{i} n_{1}(u+x, t) / \hat{c} t=-k_{2}(u+x) n_{1}(u+x, t) \\
+k_{1}(u+h+x) n_{2}(u+h+x, t)+k_{4}(u) D(t) \\
i n_{2}(u+h+x, t) / \hat{c} t=k_{2}(u+x) n_{1}(u+x, t)- \\
{\left[k_{1}(u+h+x)+k_{3}(u+h+x)\right] n_{2}(u+h+x, t)} \\
D(t)=1-\int_{-x}^{x}\left[n_{1}(u+x, t)-\right. \\
\left.n_{2}(u+h+x, t)\right] \mathrm{d} x .
\end{gathered}
$$

$D(t)$ is the number of detached cross-bridges, independent of the extension in the $\mathrm{S}_{2}$ link. Note that $k_{4}$ equals a delta-function. The force can be calculated from the cross-bridge distribution at time $t$ with

$$
\begin{aligned}
F(t)= & \int_{-x}^{x} K\left[(u+x) n_{1}(u+x, t)+\right. \\
& \left.(u+h+x) n_{2}(u+h+x, t)\right] \mathrm{d} x .
\end{aligned}
$$

In equation (6), $K$ represents the spring constant of the $S$, link. In the HS-model. $K$ equals 2.5 exp $[-4]$ and in the JSS-model $2.2 \exp [-4] \mathrm{Nm}^{-1}$.

The solution of these eytutions is rather complicated when the relative position of the filaments is a function of time.

A numerical solution can be derived by application of the basic partial differential equations of both the original models. The change of the amount of these cross-bridges in configuration state $1\left(\mathrm{~d} n_{1}\right)$ and $2\left(\mathrm{~d} n_{2}\right)$ at time $t$ can be described by

$$
\begin{gathered}
\mathrm{d} n_{1}(u+x, t)=n_{1}(u+x+\mathrm{d} x . t+\mathrm{d} t)-n_{1}(u+x, t) \\
\mathrm{d} n_{2}(u+h+x, t)=n_{2}(u+h+x+\mathrm{d} x, t+\mathrm{d} t) \\
-n_{2}(u+h+x, t) .
\end{gathered}
$$

When $\mathrm{d} t$ and $\mathrm{d} x$ are small enough the change $\mathrm{d} n_{i}$ can be approximated according to the implicit method by

$$
\mathrm{d} n_{1}=\left[\mathrm{d} n_{1}(u+x, t)+\mathrm{d} n_{1}(u+x+\mathrm{d} x, t+\mathrm{d} t)\right] / 2
$$

$\mathrm{d} n_{2}=\left[\mathrm{d} n_{2}\left(u+h+x_{1} t\right)+\mathrm{d} n_{2}\left(u+h+x+\mathrm{d} x_{1} t+\mathrm{d} t\right)\right] / 2$.

$d x$ is given by $x^{\prime}(t) \times d t$ with $x(t)$ chosen as an arbitrary length change as function of time. It follows that in the HS-model the value $n_{2}\left(u+h+x+\mathrm{d} x_{1} t+\mathrm{d} t\right)$ can be computed with

$$
\begin{aligned}
n_{2}(i+1, j+1)= & n_{2}(i, j)+\mathrm{d} t\left[k_{2}(i)+k_{2}(i+1)\right. \\
& -\left\{k_{1}(i)+k_{2}(i)\right\}_{2} n_{2}(i, j)-\left\{k_{1}(i+1)\right. \\
& \left.\left.+k_{2}(i+1)\right\} n_{2}(i+1, j+1)\right] 2
\end{aligned}
$$

and $n_{1}(u+x+d x, t+d t)$ is calculated with

$$
n_{1}(i+1, j+1)=1.0-n_{2}(i+1, j+1) .
$$

$i$ represents the extension or compression in the $S_{2}$ link; for $n_{1}$ this will be $u+x$, for $n_{2}: u+h+x$. Therefore $i+1$ represents $u+x+\mathrm{d} x$ for $n_{1}$ and $u+h$ $+x+\mathrm{d} x$ for $n_{2}$, while $j$ and $j+1$ represents the time $t$ respectively $t+\mathrm{d} t$ for both $n_{1}$ and $n_{2}$.

In the JSS-model, attachment and detachment contribute to tension recovery making the computations much more time consuming. During the time interval $\mathrm{d} t$ new cross-bridges with extension zero (i.e. $u+x$ $+\mathrm{d} x=0$ ) in the $\mathrm{S}_{2}$ link are formed in the first attached state (the $x$-position in Julian e't al., 1973, 1974). For every extension $u+i \times d x$ ( $i$ is a positive integer), the changing amount of cross-bridges must be calculated by solution of two partial differential equations.

The genera! form of these equations describing the JSS-model is

$$
\begin{aligned}
n_{1}(i+1, j+1)= & n_{1}(i, j)+\mathrm{d} t\left[k_{1}(i) n_{2}(i, j)\right. \\
& +k_{1}(i+1) n_{2}(i+1, j+1) \\
& -k_{2}(i) n_{1}(i, j) \\
& -k_{2}(i+1) n_{1}(i+1, j+1)+k_{4}(i) D(j) \\
& \left.+k_{4}(i+1) D(j+1)\right] / 2
\end{aligned}
$$




$$
\begin{aligned}
n_{2}(i+1, j+1) & =n_{2}(i, j)+\mathrm{d} t\left[k_{2}(i) n_{1}(i, j)\right. \\
& +k_{2}(i+1) n_{1}(i+1, j+1) \\
& -k_{1}(i) n_{2}(i, j)-k_{1}(i+1) n_{2}(i+1, j+1) \\
& -k_{3}(i) n_{2}(i, j) \\
& \left.-k_{3}(i+1) n_{2}(i+1, j+1)\right] / 2 \quad(1 \mathrm{lb}) \\
D(j+1)=1.0- & \sum_{m=0}^{i+1}\left[n_{1}(m, j+1)+n_{2}(m, j+1)\right] .(1 \mathrm{lc})
\end{aligned}
$$

$D, n_{1}$ and $n_{2}$ denote respectively the $D, \alpha$ - and $\beta$ position of the cross-bridges as defined by Julian et al. (1973, 1974; see also Fig. 1). The time interval used for integration was $0.01 \mathrm{~ms}$ and the corresponding relative filament displacement $x$ was between 0.01 and $1.0 \mathrm{~nm}$, dependent upon the relative filament velocity chosen. The accuracy of the calculation was checked with an integration time of $0.001 \mathrm{~ms}$. Cross-bridge distributions were compared and in cases where the largest deviation in results should appear, i.e. for maximum velocities of length change, the values of the crossbridge distribution calculated with $0.01 \mathrm{~ms}$ differed by less than 0.001 of their absolute values.

\section{RESULTS}

\section{Force transients of quick length changes}

A response of the muscle during about $100 \mathrm{~ms}$ to both lengthening and shortening is shown in Fig. 2 together with simulations of both models for com- parable amplitude and velocity of length changes. $T_{1}$ is determined as the extreme value of tension during the length change (phase 1) according to the definition of Huxley and Simmons (1971b). According to the definition given by Ford et al. (1977), $T_{2}$ is the tension at the first local extreme, or in case of an inflexion $T_{2}$ is found by backward extrapolation to the intersection with the initial fall of tension.

In the HS-model, the tension transient always reaches a horizontal plateau at the final extreme tension $T_{2}$. Two local extreme values can be detected in the recovery phase after the length change in simulations of the JSS-model dependent upon the amplitude of the length change. Indeed, the slope of the tension transient during the third phase as calculated with the JSS-model depends on the amplitude of the length change. From the literature it appears that such transients as calculated here for the JSS-model are known to be recorded in many different muscle preparations such as glycerinated single fibres (Heinl et al., 1974; Abbott and Steiger, 1977) single fibres (Julian and Sollins, 1975) and whole muscle (Stienen and Blange, 1978; Blange and Stienen, 1979).

The experimental record and the JSS-model simulation for release show a slow recovery during phase 4 which takes place in an exponential fashion. Within about $100 \mathrm{~ms}$ tension has practically reached the isometric tension value $T_{0}$. After lengthening, tension recovery is seen to take longer than $100 \mathrm{~ms}$ before the isometric tension value is reached. A full tension
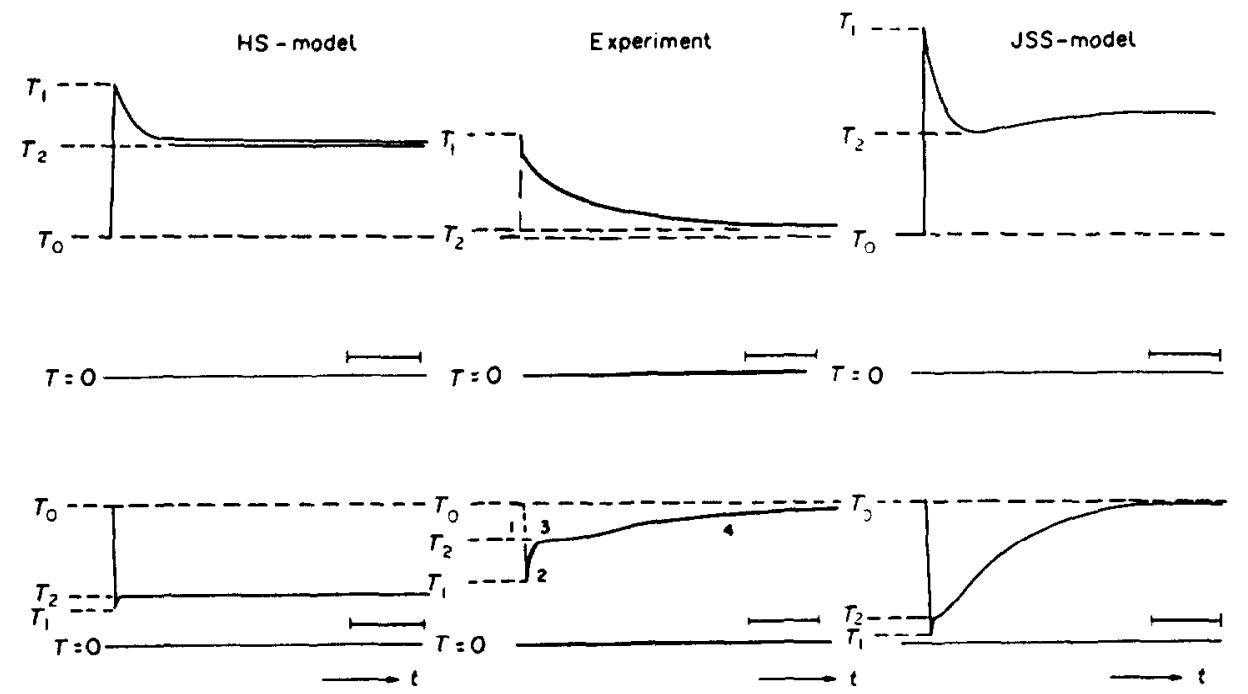

Fig. 2. A typical response to a stretch (upper) and a release (lower) performed on a sartorius muscle in an experiment and as calculated with the HS-and JSS-models. In the experiments: RFV $=8.8 \mathrm{~nm} \mathrm{~ms}^{-1}:$ RFD $=9 \mathrm{~nm}$. In the simulations: RFV $=10 \mathrm{~nm} \mathrm{~ms}^{-1} ;$ RFD $=8 \mathrm{~nm}$. Calibration bars indicate $20 \mathrm{~ms}$. As defined by Huxley and Simmons $(1971 \mathrm{~b})$ all transients show the characteristic tensions $T_{0}, T_{1}, T_{2}$ and phase of length change (1), early fast recovery phase after length change (2), plateau phase (3), and slow recovery phase (4). The HS-model simulates the response up to the second phase only, always reaching a horizontal plateau at a final extreme tension value $T_{2}$. In the JSS-model simulation of a lengthening. $T_{2}$ shows up as a local minimum value of an undershoot. The slow recovery after releases is seen both in the experiment and in the JSS-model simulation to be fulfilled within about $100 \mathrm{~ms}$ following an exponential time course. After stretch, this recovery phase lasts longer than $100 \mathrm{~ms}$ and in the JSS model simulation it was calculated to have a duration of a few seconds. 
recovery back to the isometric tension value $T_{0}$ alter a lengthening of about $10 \mathrm{~nm}$ was calculated to last a few seconds in the JSS-model.

Force responses to different length changes at a constant velocity are given at an expanded time scale in Figs 3 and 4 . Figure 3 shows four superimposed releases of which the relative filament displacements (RFD) amount to 4.4,6.6, 13.2 and $17.6 \mathrm{~nm}$ as calculated from muscle length and sarcomere length, and executed with a relative flament velocity (RFV) of length change of $8.8 \mathrm{~nm} \mathrm{~ms}^{-1}$. Computational results for both models are also depicted for releases with an RFD of 4,7 and $10 \mathrm{~nm}$ and an RFV of $10 \mathrm{~nm} \mathrm{~ms}^{-1}$. For large amplitude releases the tension-time functions show a shoulder (inflexion) in the first phase. In the experiments the time derivative of the tension transient in the first phase changes continuously while in the model simulations the time derivative is nearly
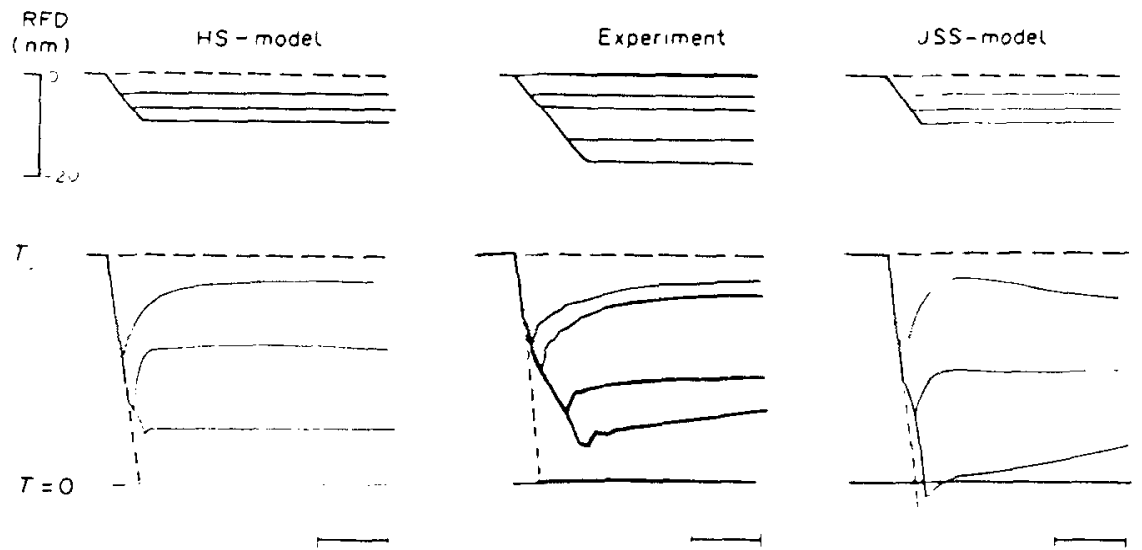

Fig. 3. Responses of different amplitudes of release at the same velocity. In the experiments: RFD = 4.4.6.6. 13.2 and $17.6 \mathrm{~nm} ; \mathrm{RFV}=8.8 \mathrm{nmms}^{-1}$. In the simulations: RFD $=4,7,10 \mathrm{~nm} ; R F V=10 \mathrm{~nm} \mathrm{~ms}^{-1}$. Calibration bar indicates $2 \mathrm{~ms}$. All transients clearly show a non-linear time course. An inflexion occurs in all transients which is more pronounced in the simulations than in the experiment. In the experiment a deviation from the approximated linear time course (dotted line) increases with the amplitude while in the simulations this deviation appears to approach a constant value. This is less conspicuous in the HS-model in which the inflexion occurs at a larger amplitude. Note that in the experimental situation the peak value of RFD is about twice as large as in the simulations.
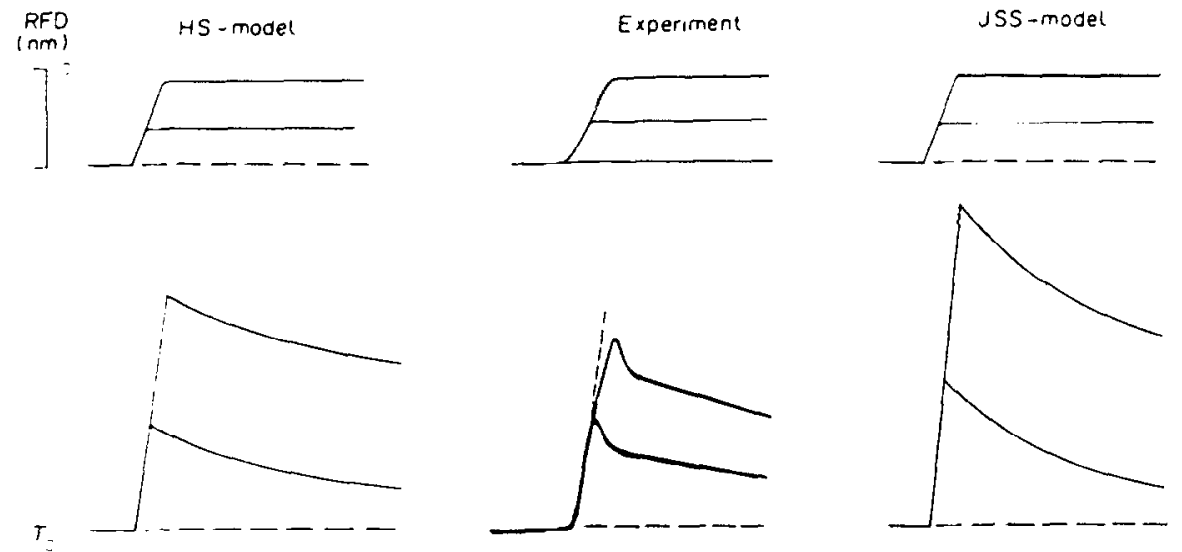
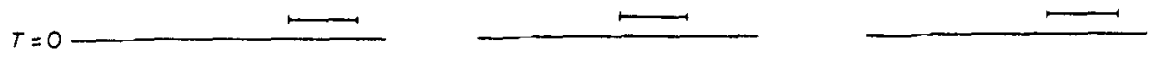

Fig. 4. Responses of different amplitudes of stretch performed at constant velocity. In the experiments: RFD $=4.4$ and $8.8 \mathrm{~nm} ; \mathrm{RFV}=13.2 \mathrm{nmms}^{-1}$. In the simulations: RFD $=4$ and $9 \mathrm{~nm} ; \mathrm{RFV}=10 \mathrm{~nm} \mathrm{~ms}^{-1}$ Calibration bar: $2 \mathrm{~ms}$. The first phase is seen to be rather linear in both model simulations. In the experiment a deviation is found showing a non-linear time course. After the stretch an initial fast cension fall is followed by a slower fall of tension. 
constant over large parts of the transient. The large change in the time derivative of the simulations occurs in a relatively small range of tension.

Figure 4 shows the experimental response of two stretches with RFD's 4.4 and $8.8 \mathrm{~nm}$ respectively performed with an RFV of $13.2 \mathrm{~nm} \mathrm{~ms}^{-1}$. The computations are given for an RFD of 4 and $9 \mathrm{~nm}$ executed with an RFV of $10 \mathrm{~nm} \mathrm{~ms}^{-1}$. An approximation of the linear time course of the tension transient as expected when no recovery takes place is depicted in the experimental figure as a dotted line. In the experiment the deviation is considerable as compared with the simulations. After lengthening, all transients obtained experimentally show an initial fast tension fall followed by a considerably slower tension recovery. This initial fast tension fall is shorter if the amplitude of stretch is larger. No such initial fast tension fall can be observed in simulations with either model.

Model simulations calculated for the typical value of RFV shown in Fig. 4 clearly result in a linear tension-time curve indicating a negligible tension recovery during lengthening.

Superimposed tension responses for different vel- ocities are given in Fig. 5 at two amplitudes to show the influence of RFV on tension recovery during release. Simulations for an RFD of 7 and of $10 \mathrm{~nm}$ are depicted for the HS-model and the JSS-model. In the experiment, these values are 7 and $14 \mathrm{~nm}$. The experimental RFV's are 4.4.8.8 and $13.2 \mathrm{~nm} \mathrm{~ms}^{-1}$ and computed RFV's are 5,10 and $20 \mathrm{~nm} \mathrm{~ms}^{-1}$. All curves look alike when the shapes of the first phase of the tension transients of both the simulations and the experiments are compared. Even at the largest RFV all transients show a similar non-linear shape. A shoulder can be observed dependent upon the amplitude and the velocity of release. When RFV is lowered a shoulder occurs at a higher tension in agreement with the single fibre results of Ford ' $a l$. (1977). Tension $T_{1}$ is seen to be decreased when the velocity of release is increased. $T_{2}$ is reached within a few ms of the end of the length change and is found to be independent of the velocity of release.

Figure 6 shows the experimental curves obtained from a stretch of $200 \mu \mathrm{m}$ (RFD is $8.8 \mathrm{~nm}$ ) executed with the same velocities as in Fig. 5 , i.e. an $\mathrm{RFV}$ of $4.4,8.8$ and $13.2 \mathrm{~nm} \mathrm{~ms}^{-1}$. Computations are
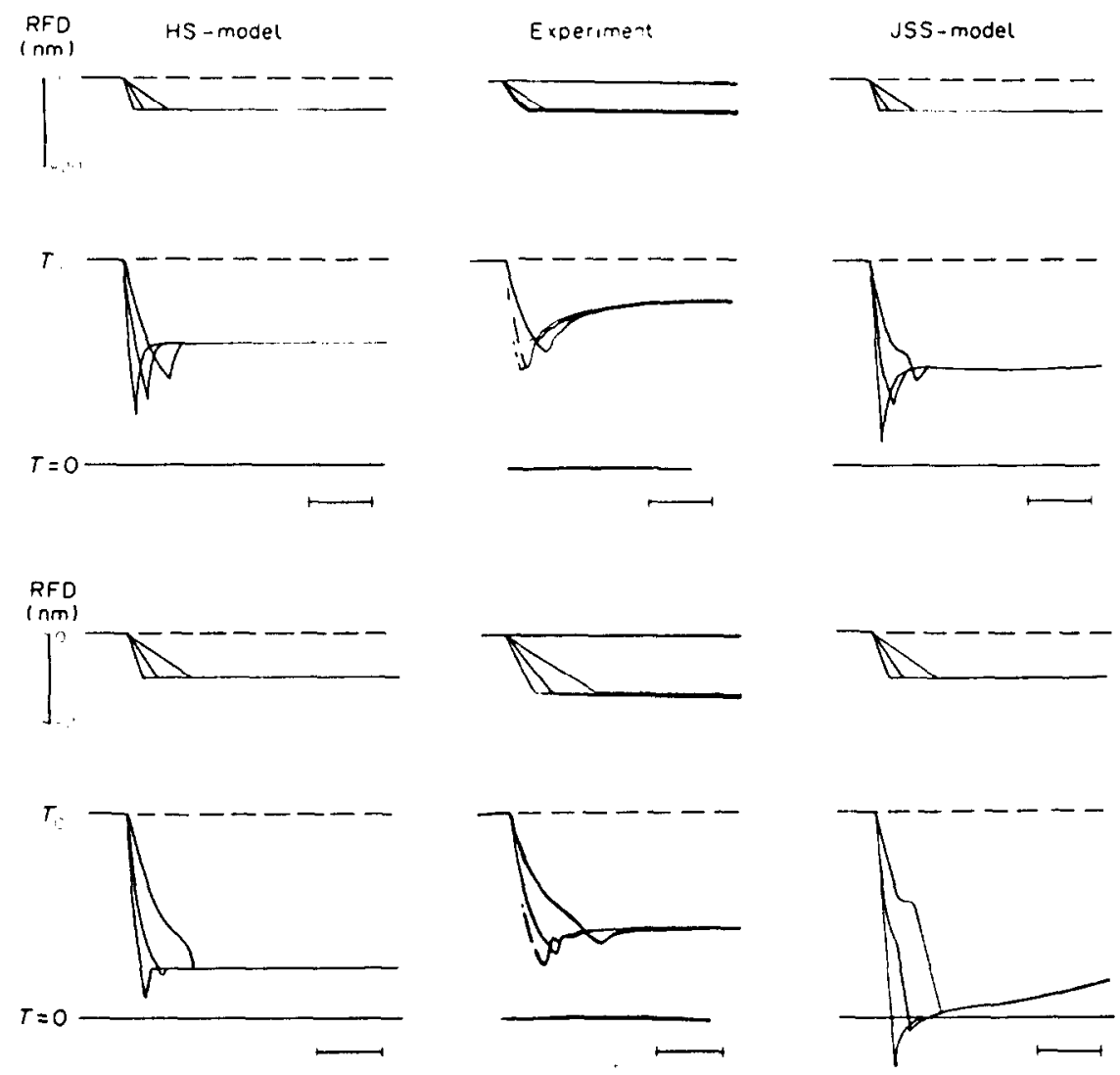

Fig. 5. Superimposed tension responses obtained at three different velocities of release at two different amplitudes. In the experiment: $R F V=4.4,8.8$ and $13.2 \mathrm{~nm} \mathrm{~ms}{ }^{-1}$; $R F D=7$ (above) and $14 \mathrm{~nm}$. In the simulations: $R F V=5,10$ and $20 \mathrm{~nm} \mathrm{~ms}^{-1} ; R F D=7$ and $10 \mathrm{~nm}$. Calibration bar: $2 \mathrm{~ms}$. The occurrence of a shoulder (inflexion) during the release is seen to be dependent of both the amplitude and of the velocity of release. For the slowest release a shoulder occurs at an RFD value of $5 \mathrm{~nm}$ for the jSS-model, at $7 \mathrm{~nm}$ for the experiment and $10 \mathrm{~nm}$ for the HS-model. When RFV is increased the shoulder occurs earlier and at a lower tension value. Note that in the experiment the peak value of amplitude is about $40 \%$ larger than in both model simulations. 

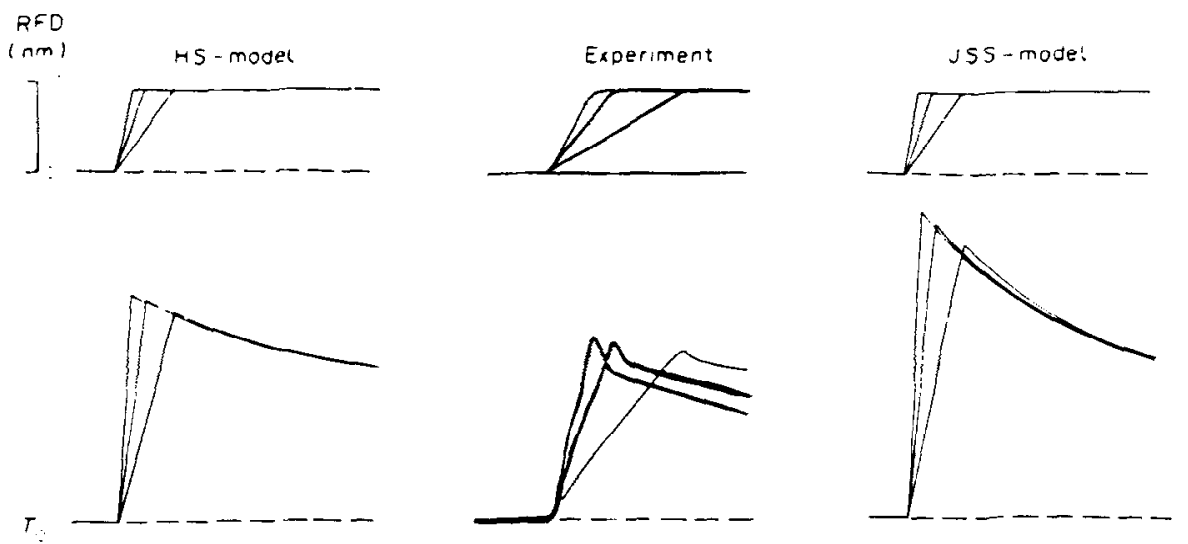

$T=0$
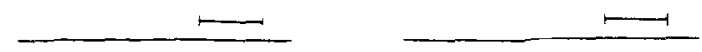

Fig. 6. Tension responses of stretches perlormed at different values of RFV. In the experiment: $R F V=4,4$. 8.8 and $13.2 \mathrm{~nm} \mathrm{~ms}^{-1} ; \mathrm{RFD}=8.8 \mathrm{~nm}$. Calibration bar indicates $1 \mathrm{~ms}$. In the simulations: $R F V=5,10$ and $20 \mathrm{~nm} \mathrm{~ms}^{-1} ;$ RFD $=9 \mathrm{~nm}$. Calibration bar indicates $2 \mathrm{~ms}$. All experimental curves show a non-linear time course while the models predict a rather linear one. Tension $T_{1}$ is seen to be decreased when RFV is decreased. After each length change a fast initial tension fall is seen for every RFV used in the experiment. The tension fall increases with the velocity RFV of stretch. After the length change the simulated curves are seen to overlap each other very early in contrast to the experimental results. Nevertheless. a tension $T_{2}$ was found in the experiment to be independent of the velocity of stretch. The experimental tension curve of the smallest velocity is retouched.
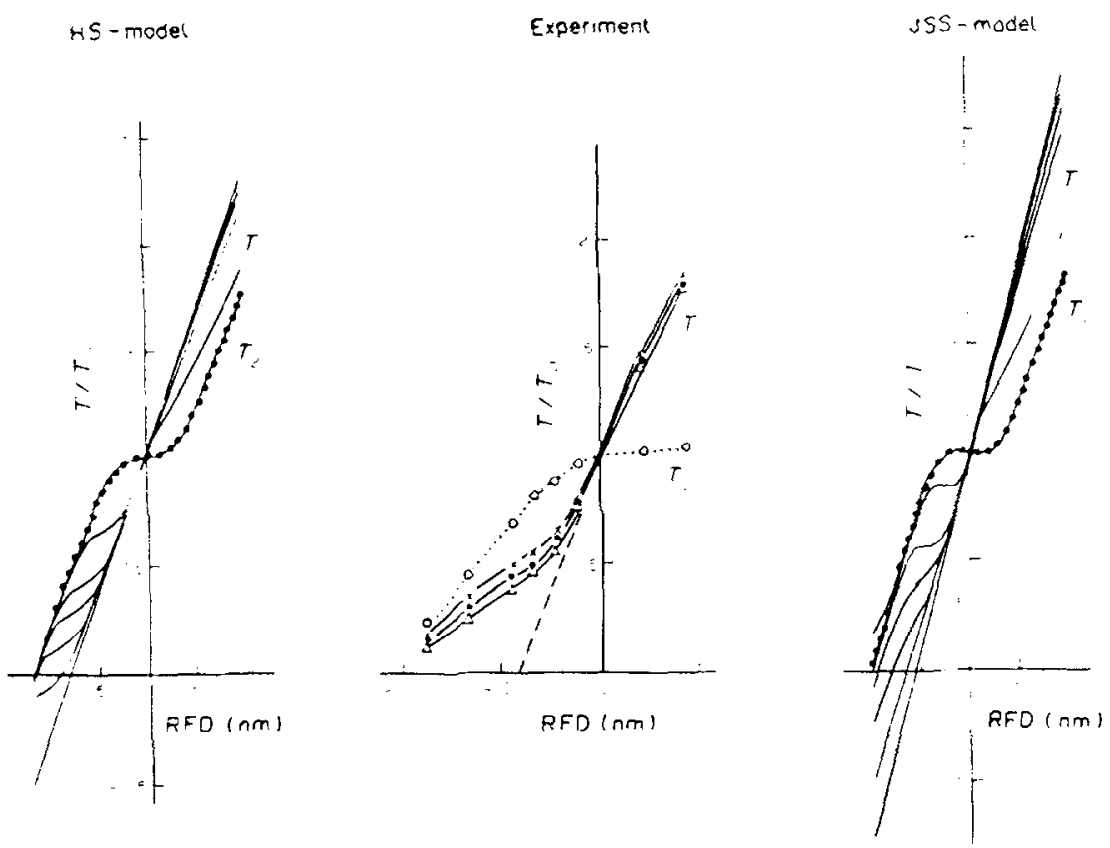

Fig. 7. Experimental and simulated $T_{1}$ and $T_{2}$ curves for different parameter values of RFV. Tensions $T_{1}$ (solid lines) and $T_{2}$ (exp $\ldots 0 \ldots 0 \ldots 0 \ldots$ sim:- $-\cdots$, ) are depicted as tensions relative to the isometric tension $T_{0}$ and as a function of the amplitude RFD (in $\mathrm{nm}$ ). In the experiment: $R F V=4.4(x), 8.8$ (.) and $13.2 \mathrm{~nm} \mathrm{~ms}^{-1}$ ( $)$.). In the simulations: RFV $=1,5,10,20,50$ and $x$ (the instantaneous $T_{1}$ curve) $\mathrm{nm} \mathrm{ms}{ }^{-1}$. For lower values of RFV the $T_{1}$ curves approach the $T_{2}$ curve at lower values of the amplitude RFD. A shoulder is seen in all curves obtained for release both in the experiment and in the simulations of both models. In the JSS-model it is seen that tension $T_{1}$ for RFV $=1 \mathrm{~nm} \mathrm{~ms}{ }^{-1}$ exceeds the $T_{2}$ curve when the amplitude of release is larger than $7 \mathrm{~nm}$. For larger values of RFD performed at this velocity (or lower) no tension $T_{2}$ can be detected. This also means that $T_{2}$ is only independent of RFV for at least moderate values. For stretches a shoulder shape is revealed only in the $T_{1}$ curve calculated for RFV $=1 \mathrm{nmms} \mathrm{ms}^{-1}$. The instantaneous $T_{1}$ curve (dashed line) in the experimental figure is drawn by eye as the initial tangent to the $T_{1}$ curve depicted for RFV $=13.2 \mathrm{~nm} \mathrm{~ms}^{-1}$. The stopes of the instantaneous $T_{1}$ curve and the final slope of the $T_{2}$ curve are seen to be different in the experiment and similar in the simulations. 
carried out for a stretch with an RFD of $9 \mathrm{~nm}$ and an RFV of 5, 10 and $20 \mathrm{~nm} \mathrm{~ms}^{-1}$ isimilar to Fig. 4). The experimental records show a non-linear tension-time course during the length change for each velocity used. Tension $T_{1}$ is found to be larger when the RFV of stretch is increased. As in Fig. 4, a fast initial tension fall can also be observed after each of these length changes. When RFV is decreased this fall in tension is smaller.

The second phase was found to last much longer after stretch than after release. Although it cannot be seen in Fig. 6. $T_{2}$ was also found to be independent of the velocity of stretch.

$T_{1}$ and $T_{2}$ curves derived from the experiments and the simulations are shown in Fig. 7 for different values of RFV. The abscissa of the $T_{1}$ curve is scaled with $\mathrm{RFD}=\mathrm{RFV} \times \mathrm{t}$. Model simulation curves are depicted for the following values of RFV: 1, 5, 10, 20, 50 and $\infty \mathrm{nm} \mathrm{ms}^{-1}$. The experimental curves are given for an RFV of $4.4,8.8$ and $13.2 \mathrm{~nm} \mathrm{~ms}^{-1}$. Because the velocity of length change is kept constant in the model simulations, the shape of the $T_{1}$ curves is the same as of the first phase of the tension transient. In the experiment as well as in the model simulations for smaller velocities of release, a shoulder (inflexion) occurs in the $T_{1}$ curve at lower amplitudes. After the shoulder the $T_{1}$ curve approaches the $T_{2}$ curve assymptotically in the HS-model calculation. In the JSS-model simulation this is only the case if RFV is at least moderate. However, when RFV amounts to $1 \mathrm{~nm} \mathrm{~ms}^{-1}$ the $T_{1}$ curve exceeds the $T_{2}$ curve if the amplitude of release is larger than about $7 \mathrm{~nm}$. The calculated $T_{1}$ curve for $R F V=1 \mathrm{~nm} \mathrm{~ms}^{-1}$ is seen to be non-linear for stretches in contrast with the $T_{1}$ curves calculated for larger values of RFV.

For the instantaneous situation both models show a linear relationship between tension $T_{1}$ and the amplitude RFD. An estimation of its slope is made in the experimental figure by drawing a tangent to the $T_{1}$ curve at the largest RFV (see the dashed line in Fig. 7).

A difference between model simulation and experiment can be seen with respect to the slope of the instantaneous $T_{1}$ curve and the tinal slope of the $T_{2}$ curve. In both model simulations these slopes become the same for large values of RFD irrespective of the direction of the length change. In the experimental result for shortening the slope of the approximated
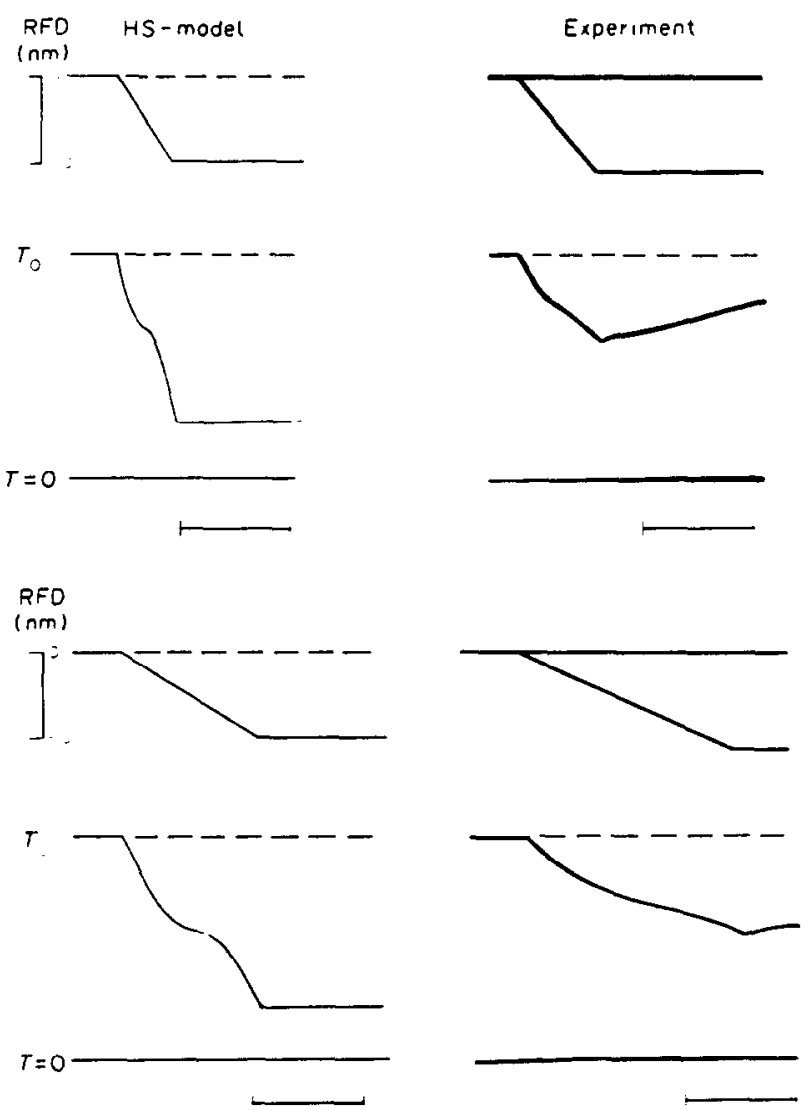
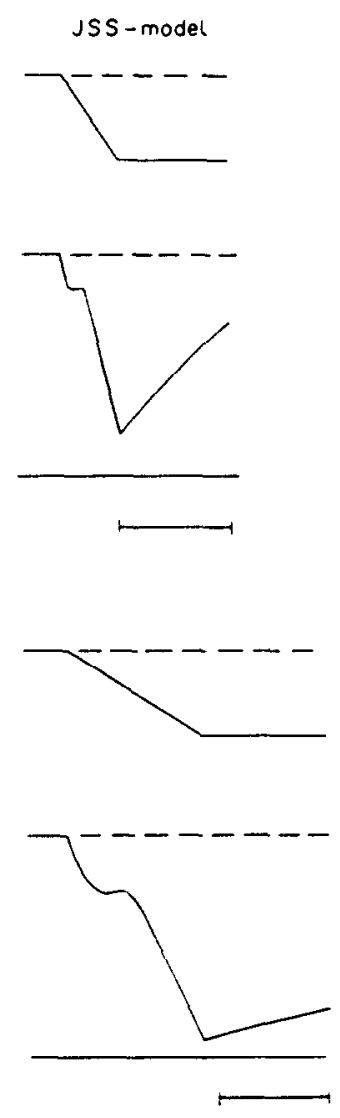

Fig. 8. Tension responses of very slow releases. In the experiment: RFV $=0.7$ (above) and $1.4 \mathrm{~nm} \mathrm{~ms}^{-1}$; RFD $=11 \mathrm{~nm}$. In the simulations: $R F V=1.0$ (above) and $2.0 \mathrm{~nm} \mathrm{~ms}^{-1} ; R F D=10 \mathrm{~nm}$. Calibration bars indicate $20 \mathrm{~ms}$ (upper half) and $4 \mathrm{~ms}$ (lower half). The shoulder configuration becomes more conspicuous for lower rates. A very small tension recovery contribution is seen in the second phase indicating a fast recovery which is aiready finished during the length change in the first phase. In the simulations the steepest slopes before and after the shoulder are seen to be equal while in the experiments this seems not the case. 
instantaneous $T_{1}$ curve (dashed line) and the final slope of the $T_{2}$ curve are quite different.

Force transients of slow releases

Figure 8 shows the experimental response of a shortening in which the RFD is $11 \mathrm{~nm}$ performed with an RFV of 1.4 and of $0.7 \mathrm{~nm} \mathrm{~ms}^{-1}$. Simulations are calculated for a release of $\mathrm{RFD}=10 \mathrm{~nm}$ performed with a velocity of $R F V=1 \mathrm{~nm} \mathrm{~ms}^{-1}$ (upper half of figure) and of $2 \mathrm{~nm} \mathrm{~ms}^{-1}$. It is seen that a shoulder exists for every velocity of length change in the first phase of all responses. For reasons mentioned before, the first phase of these transients can roughly be considered as the $T_{1}$ curve belonging to a given parameter value of RFV. An approximation of the final slope of the $T_{2}$ curve can be the steepest slope of the transient after the shoulder. This approximation is justified from that moment where the early fast recovery is already completely finished during the first phase and as long as the slow recovery has a negligible influence on tension recovery during the length change. It can be observed that this is most probably the case in all figures showing a very small attribution of tension fast recovery in the second phase followed by a slight increase of tension in the third phase. It can be seen that the steepest slopes before and after the shoulder are equal in the simulations. In the experiments this seems not to be the case. This is conceivable, however, in view of the differences between the slope of the $T_{1}$ curve and the final slope of the $T_{2}$ curve (c.f. Fig. 7).

\section{DISCUSSION}

The aim of our study was to investigate the influence of the velocity of length change on the tension transients in muscle tissue. The conspicuous effects mainly occur in the early phases of the tension transients. The force during these phases is totally (HSmodel) or mainly (JSS-model) determined by crossbridge transitions between different attached states. The typical features of the calculated tension transients obtained during length changes of constant velocity can be qualitatively summarized as follows:

1. During a steady shortening both model calculations reveal a point of inflexion $\left(\hat{c}^{-2} T / \mathrm{c}^{2}=0\right)$ in the tension transients. When the velocity of length change is increased the tension at which this point of inflexion occurs is lower (Ford el al., 1977) and the inflexion appears earlier. As a consequence the $T_{1}$ curves calculated for different shortenings at a constant velocity also show a point of inflexion $\left(\hat{c}^{2} T / \hat{c} u^{2}=0\right)$.

In the HS-model the amplitude of length change (RFD) at which the point of inflexion occurs in the $T_{1}$ curve increases with the velocity of length change (RFV).

In the JSS model the value of RFD at the point of inflexion increases too with the velocity of length change up to about $5 \mathrm{~nm}$. From then on this value increases considerably less with RFV and even seems to be rather independent of it.
A point of inflexion occurs only during rather slow stretches (RFV is $1 \mathrm{~nm} \mathrm{~ms}^{-1}$ ).

2. The slope of the tension transients for releases at the point of inflexion decreases with increasing velocity of length change in both models. As a consequence the transient and the $T_{1}$ curve calculated for a larger velocity are rounded off more.

3. For both models $T_{2}$ is found to be independent of the velocity of length change.

A comparison with the experimental results reveals the following:

1. In the experimental records the inflexion shows up clearly in slow releases with a duration of about $3 \mathrm{~ms}$ and more. In faster releases the occurrence of an inflexion cannot be unambiguously established.

2. The experimental force records and $T_{1}$ curves show that the inflexion rounds off when the shortening is performed at a higher velocity.

3. In the release experiments the tension change during the fast recovery phase. after the shortening, decreases with a decreasing velocity of length change.

4. Both in stretch and in release the final slope of the $T_{2}$ curve is less steep than the slope of the approximated instantaneous $T_{1}$ curve. $T_{2}$ determined experimentally is rather independent of the amplitude of stretch.

The experimental results presented here from whole muscle preparations agree rather well with the results obtained in experiments on single fibres with respect to steady releases as presented by Ford $t t a l .(1977)$ and with respect to steady stretches as presented by Edman et al. (1981). It is noted by Ford et al. (1977) that a point of inflexion occurs during relatively slow releases. The experimental results presented here show features which correspond with the results of Ford et al. (1977, Fig. 29A) in the range covered. This correspondence with single fibre results is remarkable because it is influenced by non-linear behaviour of the tendons and by nonuniform distribution of compliance in the whole muscle. Stienen and Blange (1981) obtained sarcomere displacement values from local movement experiments using markers along the muscle surface. $T_{1}$ and $T_{2}$ curves from these measurements were seen to be shifted to lower displacement values. while at the same time representing the results of a somewhat lower velocity of length change. The shapes of the $T_{1}$ and $T_{2}$ curves, however, are not affected.

In the experiments of Ford te al. $(1977,1981)$ displacements up to $6 \mathrm{~nm}$ per half sarcomere were made. When considering the first phase in their transients (Ford et al., 1977, Figs 12 and 14; 1981, Figs 5 and 6$)$ it is seen that in all cases a non-linearity is clearly detectable. A point of inflexion however is not found which could be attributed to the small amplitude and high velocity of the length change.

Large stretches were performed with a low constant velocity by Edman et al. (1981, Fig. 1). A clear nonlinear deviation can be detected during the first phase despite the small time scale.

All transients showed a fast initial tension recovery 
after the stretch followed by a slower one. The initial tension fall increases with the velocity at which the lengthening is performed. similar to the fast recovery after releases. The non-uniform distribution of compliance tends to give rise to an overshoot in the local length change. However, the estimate of Stienen and Blangé (1981) indicates that this effect is too small to totally explain the early rapid recovery. This is determined by a mixture of distinctly different rate constants. $T_{2}$ needs to be revised for stretch in which the slower component contributes.

\section{Model interpretations of experimental results}

An inflexion is seen during the first phase in the experimental and simulated releases. It can be explained from the models as follows. During shortening the rate constant $k_{2}$ considerably increases compared with $k_{1}$. So the dynamic equilibrium shifts towards the configuration state $n_{2}$ resulting in an extra tension to partly balance the loss of tension as predicted by the instantaneous curve. When velocity of length change is smaller, the duration of the length change is larger and extra tension generation nullifies or even exceeds the instantaneous loss of tension due to shortening. The contribution of the extra tension generation increases with the exponential increase of the rate constant $k_{2}$. The contribution of the other rate constants $\left(k_{1}\right.$ and $k_{4}$ in the JSS-model) is negligible during the early recovery phase. The progressive increase in the value of $k_{2}$ with the amplitude of release causes the amount of cross-bridges in the attached state $n_{1}$ to decrease during shortening. The point of inflexion follows from the equilibrium between the increase of tension due to the increase of $k_{2}$ and the decrease of tension due to decrease of $n_{1}$. For stretches the same reasoning can be applied but in the opposite direction. In this case $k_{2}$ becomes negligible with respect to the other rate constants $\left(k_{1}, k_{3}\right.$ and $k_{4}$ for the JSS-model).

An inflexion is seen for all shortening velocities in the model results. The inflexion is more pronounced in the simulations with the JSS-model than with the HSmodel. The time derivative at the point of inflexion is larger. Of course it is possible by changing the rate constants for transition between different attached states to fit the simulation with the experiments. However, as a result, the fast recovery during phase 2 is then slowed down which would not be realistic as indicated by Huxley and Simmons (197/b) and by Julian et al. (1974). Also when Fig. 2 is considered, the rate of tension development is seen to be of the right order of magnitude.

Another explanation is provided by the assumption of more attached states (Huxley and Simmons. 1971 b; Huxley, 1980). The inflexion is also rounded off when the rates of redistribution between the different states are high. A further discrepancy can be observed in the final slope of the $T_{1}$ curve and the slope of the approximated instantaneous $T_{1}$ curve. When the point of inflexion is interpreted as a redistribution phenomenon between the attached states, and if the stiffness of the $S_{2}$ link in the cross-bridge is the same in both states, then the initial slope of the instantaneous $T_{1}$ curve and the final slope of the $T_{2}$ curve have to be equal. This is not observed. A possible explanation for the different slopes found experimentally is that the $T_{2}$ curve is influenced by re-attachment. This view is compatible with the fast detachment of cross-bridges after release which explains the stiffness measured during the transients (Stienen and Blangé, 1980, 1981). When the shortening is slower a larger contribution of the slow recovery can also be expected. The contribution of the slow recovery must however be overestimated to explain the discrepancy in the slopes of the $T_{2}$ curve and the instantaneous $T_{1}$ curve. It is well known that the slow recovery is exponential (Julian et al. 1974; Stienen and Blange, 1980). The time constant which dominates the slow recovery is rather independent of the amplitude of the length change and amounts to about $40 \mathrm{~ms}$ at $0^{\circ} \mathrm{C}$. When the length changes are performed within about $5 \mathrm{~ms}$ the contribution of the force development due to slow recovery is maximally about $10{ }^{\prime \prime}$, of the deviation of the tension at $\iota=\propto\left(T_{0}\right.$ in this case). The discrepancy between the slopes of the $T_{2}$ curve and the instantancous $T_{1}$ curve is considerably larger.

As suggested by Hill and Eisenberg (1979) this discrepancy could be resolved by the assumption of different stiffness values for each state. However under this assumption the instantaneous $T_{1}$ curve (as approximated) gives the weighted mean stiffness for the different attached states while the $T_{2}$ curve gives information about the stiffness for state 2 (release) and state 1 (stretch). Assuming a model with three attached states with a specific stiffness value in each attached state, it will be possible to obtain three different slopes for the instantaneous curve, the $T_{2}$ curve for release and the $T_{2}$ curve for stretch. Such a model suits the observations with respect to the $T_{2}$ curve and the instantaneous $T_{1}$ curve.

With both models the point of inflexion also should occur by lengthening due to the differences in rate constants. Since the rate constant $k_{2}$ decreases very slowly ( $k_{1}$ and $k_{3}$ stay constant) the equilibrium is much less disturbed and extra tension degeneration becomes visible only during the very slow releases. As seen in the model simulations, a clear point of inflexion occurs after about $6 \mathrm{~ms}$ only for an RFV of $1 \mathrm{~nm} \mathrm{~ms}^{-1}$. In the HS-model tension decreases only due to transition to state $n_{1}$ but in the JSS-model a larger decrease of tension is attributed to detachment.

The intersection of the instantaneous $T_{1}$ curve with the RFD-axis is determined by the stretch $u$ in the S2 link in state $n_{1}$ in the isometric situation, the crossbridge length $h$, and the actual distribution of crossbridges over the attached states $n_{1}$ and $n_{2}$. This intersection is $\mathbf{8 ~ n m}$ for the HS-model calculation and $6 \mathrm{~nm}$ for the JSS-model. This value is found to be $4 \mathrm{~nm}$ or less in the single fibre experiments of Ford et al. (1977). This difference can be solved by changing the model value for $h$ or by the rate constant values 
governing the cross-bridge distribution ratio in the isometric tetanic situation.

Achnowiedigements-This work is supported by the Netherlands Organisation for the Advancement of Puru Research (Z.W.O). We gratefully acknowledge $H$. van der Vorst (ACCU) for his mathematical sunport, P. Schiereck. P. Kil and A. Crowe for their fruitful discussion during this investigation.

\section{REFERENCES}

Abbott, R. H. and Steiger, G. J. (1977) Temperature and amplitude dependence of tension transients in glycerinated skeletal and insect fibrillar muscle. J. Physiol. 266, 13-42.

Blangé. T. Karemaker. J. M. and Kramer, A. E. J. J. (1972) Elasticity as an expression of cross-bridge activity in rat muscle. Pfügers Arch. ges Physiol. 336, 277-288.

Blangé. T. and Stienen, G. J. M. (1979) Isometric tension transients in skeletal muscle before and after inhibition of ATP synthesis. The Role of Cross-bridges in Muscle Contraction (Edited by Sugi. H. and Pollack, G. H.), p. 342. Tokyo University Press.

De Beer, E. L., Blangé. T. Van Kaam. F. A. M. and Stienen, G. J. M. (1982) The effect of the rate of length change on $T$ : and $T 2$ curves of frog skeletal muscle. J. Physiol. 330, 44.

Edman, K. A. P., Elzinga. G. and Noble, M. I. M. (1981) Critical sarcomere extension required to recruit a decaying component of extra force during stretch in tetanic contractions of frog skeletal muscle fibres. J. gen. Physiol. 78, 365-382.

Ford. L. E., Huxley. A. F. and Simmons, R. M. (1977) Tension responses to sudden length change in stimulated frog muscle fibres near slack length. $J$. Physiol. 269, 441-515.

Ford, L. E. Huxley, A. F. and Simmons. R. M. (1981) The relation between stiffness and filament overlap in stimulated frog muscle fibres. J. Physiol. 311, 219-249.

Gordon, A. M., Huxlcy, A. F. and Julian, F. J. (1966a) Tension development in highly stretched vertebrate muscle fibres. $J$. Physiol 184, 143-169.

Gordon, A. M., Huxley, A. F. and Julian, F. J. (1966b) The variation in isometric tension with sarcomere length in vertebrate muscle fibres. J. Physiol. 237, 170-192.

Hanson, J. and Huxley, H. E. (1953) Structural basis of the cross striations in muscle. Nature, Lond. 172, 530-532.

Heinl, P., Kuhn, H. J. and Ruegg, J. C. (1974) Tension responses to quick length changes of glycerinated skeletal muscle fibres from the frog and tortoise. J. Physiol. 237. 243-258

Hill, T. L. and Eisenberg, E. (1979) Simplified theory of the Huxley-Simmons T0, T1 and T2 in muscle models with two attached states. The Role of Cross-bridges in Muscle Contraction (Edited by Sugi, H. and Pollack, G. H.). p. 541 . Tokyo University Press.
Huxley, A. F. (1957) Muscle structure and theories of contraction. Proy. Biophys. (ftem. 7. 255-318.

Huxley, A. F. (1980) The mechanical properties of crossbridges and their relation to muscle contraction. Adtanct's in Physiological Scienci' Vol. 5. Molecular and Collular Aspects of Muscle Function (Edited by Varga. E.. Kover. A. and Kovacs. L.) pp. 1-12.

Huxley, A. F. and Niedergerke. R. (1954) Interference microscopy of living muscle fibres. Niuture. Lond. 173 , 971-973.

Huxley, A. F. and Simmons, R. M. (1971a) Mechanical properties of the cross-bridges of frog striated muscle. $J$. Physiol. 218, 59.

Huxley, A. F. and Simmons. R. M. (1971b) Proposed mechanism of force generation in striated muscle. Nature', Lond. 233, 533-538.

Huxley, A. F. and Simmons, R. M. (1973) Mechanical transients and the origin of muscular force. Cold Spring Harb. Symp. quant. Biol. 37, 669-680.

Huxley, H. E. (1953) Electron microscope studies of the organisation of the filaments in striated muscle. Biochim. biophys. Acta. 12, 387-394.

Huxley, H. E. (1971) The structural basis of muscular contraction. Proc. R. Soc. London. B178, 131-149.

Julian, F. J. and Sollins, M. R. (1975) Variation of muscle stiflness with force at increasing speeds of shortening. $J$. gen. Physiol. 66. 287-302.

Julian. F. J., Sollins, K. R. and Sollins, M. R. (1973) A model for muscle contraction in which cross-bridge attachment and force generation are distinct. Cold Spring Hurb. Symp. quant. Biol. 37, 685-688.

Julian, F. J., Sollins, K. R. and Sollins, M. R. (1974) A model for the transient and steady-state mechanical behavior of contracting muscle. Biophys. J. 14, 546-562.

Podolsky, R. J. (1960) Kinctics of muscular contraction: the approach to the steady-state. Nature, Lond. 188, 666.668.

Podolsky, R. J. and Nolan, A. C. (1973) Muscle contraction transients, cross-bridge kinetics and the Fenn effect. Cold Spring Harb. Symp. quant, Biol. 37, 661-668.

Ramsey, R. W. and Street. S. F. (1940) The isometric length-tension diagram of isolated skeletal muscle fibres of the frog. J. cell comp. Physiot. 15, 11-34.

Stienen. G. J. M. (1981) The dynamic properties of the contractile mechanism in skeletal muscie. Thesis, University of Amsierdam.

Stienen, G. J. M. and Blangé, T. (1980) A quantitative analysis of the force transients of skeletal muscle in response to quick changes in length. Cardia Dynamics (Edited by Baan, J., Arntzenius, A. C. and Yellin. E. L.) pp. 69-78.

Stienen, G. J. M. and Blange. T. (1981) Local movement in stimulated frog sartorius muscle. J. ge'n. Phisiol 78, $151-170$

Stienen, G. J. M., Blangé, T. and Schnerr, M. C. (1978) Tension responses of frog sartorius muscle to quick ramp shaped shortenings and some effects of metabolic inhibition. Pffügers Arch. ges. Physiol. 376, 97-104. 